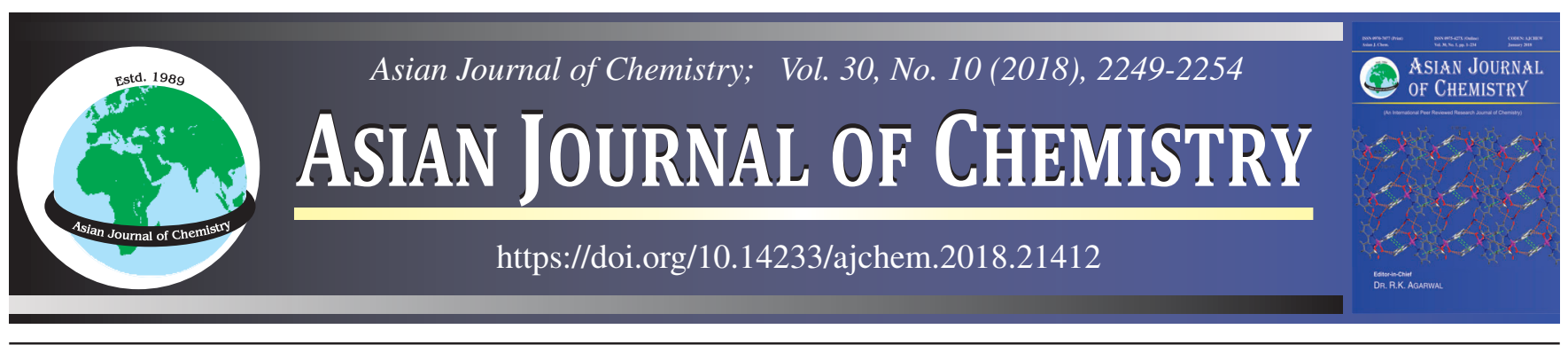

\title{
Efficiency of X-PIPS Si(Li) Detector and Bremsstrahlung Spectra in Thick Target Produced by $\beta$-Emitter ${ }^{45} \mathrm{Ca}$
}

\section{TAJINDER Singh}

Department of Physics, Mata Gujri College, Fatehgarh Sahib-140 406, India

Corresponding author: E-mail: tajindersingh2k9@gmail.com

The complete response of the X-PIPS Si(Li) detector has been developed to calculate the total bremsstrahlung spectral photon distributions in thick metallic targets $(\mathrm{Al}, \mathrm{Cu}, \mathrm{Sn}$ and $\mathrm{Pb})$ produced by continuous $\beta$-particles of ${ }^{45} \mathrm{Ca}$ in the photon energy region of 5 to $30 \mathrm{keV}$. The geometrical full-energy peak detection efficiency of the X-PIPS $\mathrm{Si}(\mathrm{Li})$ detector has been determined in the photon energy region 5-30 $\mathrm{keV}$. A $\gamma$-ray source of ${ }^{133} \mathrm{Ba}$ and the X-rays peaks of $\mathrm{Ti}, \mathrm{Cu}$, Mo and $\mathrm{Sn}$ materials were used for determining the detector parameters and for calibration of the detector. The values of photo-fractions were determined at different photon energies by using the X-rays peaks | produced at different photon energy. The geometrical full-energy peak detection efficiency of the detector was determined by using the | values of the photo-fraction and intrinsic efficiency of the X-PIPS detector. It has been found that the efficiency of detector efficiency is varying from 95 to $5 \%$ in the studied photon energy region. It is observed that the experimental results show better agreement with the modified Elwert factor (relativistic) Bethe-Heitler theory, which includes the polarization bremsstrahlung into ordinary bremsstrahlung by using stripped atom approximation.

Keywords: X-PIPS Si(Li) detector, Total Bremsstrahlung, Polarization, Ordinary Bremsstrahlung.

ᄂ - - - - - - - - - - - - - - - - - - - - - - - - - - - - -

\section{INTRODUCTION}

The measurements of bremsstrahlung spectral photon energy distributions produced by continuous beta particles in thick targets, particularly at low and medium photon energy region of 5-30 keV, requires a high resolution $\mathrm{Si}(\mathrm{Li})$ detector. The earlier measurements of bremsstrahlung [1,2] were made with the ionization chamber, proportional counters and G.M. Counters. Later, various researchers $[3,4]$ have used the $\mathrm{NaI}$ (Tl) detectors having high efficiency and more effective to make the measurements of bremsstrahlung at high energies, particularly above photon energy of $30 \mathrm{keV}$. Numbers of measurements [5-7] were also reported below $30 \mathrm{keV}$ by using $\mathrm{Si}(\mathrm{Li})$ detector. In the present measurement, the complete response of the detector taken into account, while measuring the bremsstrahlung spectra. The present measurements were taken to obtain the total bremsstrahlung (BS) spectral photon distribution, produced by the continuous $\beta$-particles of ${ }^{45} \mathrm{Ca}$ in thick metallic targets of $\mathrm{Al}, \mathrm{Cu}, \mathrm{Sn}$ and $\mathrm{Pb}$, in the photon energy regions of 5-30 keV. So, in view of the range of photon energy region a high efficiency and high resolution $\mathrm{Si}(\mathrm{Li})$ detector X-PIPS have been used in the present measurements.

X-PIPS $\mathrm{Si}(\mathrm{Li})$ detector is a compact system with a PIPS (passivated implanted planar silicon) detector. X-PIPS Si(Li) detector (Canberra make) is sensitive to ionizing radiation, particularly X-rays and low energy gamma rays. The detector is more suitable for the study of bremsstrahlung spectra owing to its high intrinsic efficiency ( $97 \%$ to $15 \%$ ) and high resolution ( $\leq 190 \mathrm{eV}$ at $5.9 \mathrm{keV}$ photon energy), in the photon energy from 5 to $30 \mathrm{keV}$. A high resolution X-PIPS (Canberra makes) $\mathrm{Si}(\mathrm{Li})$ detector has been shielded with a Lead bricks and is further connected to the power supply (ORTEC make), spectroscopy amplifier (ORTEC makes) and multichannel analyzer (ORTEC makes) with Maestro 32 software, was used to measure the bremsstrahlung spectral photon distributions in metallic targets produced by continuous $\beta$-particles in the photon energy region of 5-30 keV. The X-PIPS Si(Li) detector has a thermo-electric cooler (TEC), which act as a heat pump and is cooling down the X-PIPS detector. The thickness of X-PIPS $\mathrm{Si}(\mathrm{Li})$ detector chip is $8 \mathrm{~mm}^{2} \times 500 \mu \mathrm{m}$. This detector has a

This is an open access journal, and articles are distributed under the terms of the Creative Commons Attribution-NonCommercial 4.0 International (CC BY-NC 4.0) License, which allows others to copy and redistribute the material in any medium or format, remix, transform, and build upon the material, as long as appropriate credit is given and the new creations are licensed under the identical terms. 
silicon chip of thickness $500 \mu \mathrm{m}$, beryllium (Be) window of thickness $25 \mu \mathrm{m}$, preamplifier, HV bias supply, Peltier cooler and temperature control system. A collimator of width $1.1 \mathrm{~mm}$ is embedded in the detector. Various detector parameters such as energy resolution, photo-fraction and detector efficiency needed for the exact response of the detector were needed to calculate the bremsstrahlung spectra.

The details about the calculation of bremsstrahlung, which include the contribution of polarization bremsstrahlung (PB) into ordinary bremsstrahlung $(\mathrm{OB})$ have been given by reported earlier [8-11]. In the present measurements, experimental results were compared with the Elwert corrected (non relativistic) Bethe-Heitler [EBH] theory, modified Elwert factor (relativistic) Bethe-Heitler $\left[\mathrm{F}_{\bmod } \mathrm{BH}\right]$ theory for ordinary bremsstrahlung and the modified Elwert factor (relativistic) Bethe-Heitler $\left[\mathrm{F}_{\bmod } \mathrm{BH}+\mathrm{PB}\right]$ theory, which include the polarization bremsstrahlung into ordinary bremsstrahlung by using stripped atom approximation (SAA) to describe the bremsstrahlung given by Avdonina and Pratt [11].

In the case of thick target, Bethe and Heitler [12] gave an expression for the bremsstrahlung spectral distribution $\mathrm{n}\left(\mathrm{W}_{\mathrm{e}}{ }^{\prime}, \mathrm{k}, \mathrm{Z}\right)$ in a sufficiently thick target to absorb an electron of energy $\mathrm{W}_{\mathrm{e}}^{\prime}$ with $\mathrm{N}$ atoms per unit volume. At lower photon energies in thick targets, Semaan and Quarles [13] have reported that the correction for the self absorption of bremsstrahlung photons in the target and electron backscattering are required for $\mathrm{n}\left(\mathrm{W}_{\mathrm{e}}{ }^{\prime}, \mathrm{k}, \mathrm{Z}\right)$. In the present measurements absorption of photons in the Be window and in Si chip is also incorporated to obtain the best accuracy. The bremsstrahlung spectral distribution $\left[\mathrm{n}_{\mathrm{cor}}\left(\mathrm{W}_{\mathrm{e}}{ }^{\prime}, \mathrm{k}, \mathrm{Z}\right)\right]$ after absorption correction and electron backscattering correction in thick target is given by:

$$
\begin{aligned}
\mathrm{n}_{\text {cor }}\left(\mathrm{W}_{\mathrm{e}}^{\prime}, \mathrm{k}, \mathrm{Z}\right)= & \mathrm{RN} \int_{1+\mathrm{k}}^{\mathrm{W}_{\mathrm{e}}^{\prime}} \frac{\mathrm{d} \sigma\left(\mathrm{W}_{\mathrm{e}}, \mathrm{k}, \mathrm{Z}\right) / \mathrm{dk}}{\left(-\mathrm{dW}_{\mathrm{e}} / \mathrm{dx}\right)} \mathrm{dW}_{\mathrm{e}} \times \exp (-\mu \mathrm{x}) \times \\
& \exp \left(-\mu_{\mathrm{Be}} \mathrm{x}_{\mathrm{Be}}\right) \times \exp \left(-\mu_{\mathrm{Si}} \mathrm{x}_{\mathrm{si}}\right)
\end{aligned}
$$

Here $\mathrm{d} \sigma\left(\mathrm{W}_{\mathrm{e}}, \mathrm{k}, \mathrm{Z}\right) / \mathrm{dk}$ is the singly differential cross section taken from the different theoretical models, ' $-\mathrm{dW}_{\mathrm{e}} / \mathrm{dx}$ ' is the total energy loss per unit path length of an electron in a target material taken from the tabulations given by Berger and Seltzer [14]. Where $\exp (-\mu \mathrm{x})$ is the absorption factor, $\mathrm{m}$ is the mass attenuation coefficient for the given target element and taken from the tabulations given by Chantler et al. [15] and ' $\mathrm{x}$ ' is the optimum thickness of the target which is equal to the range of the beta particle in a target. Where $\mathrm{x}_{\mathrm{Be}}$ is the thickness of Be window and $\mathrm{X}_{\mathrm{Si}}$ is the thickness of silicon chip of the detector. ' $\mathrm{R}$ ' is the electron backscattering factor [13].

The bremsstrahlung spectral distribution in a thick target obtained on complete absorption of $\beta$-particles of endpoint energy $\mathrm{W}_{\max }$ is expressed as a number of photons of energy $\mathrm{k}$ per unit $m_{0} c^{2}$ per $\beta$-disintegration for continuous $\beta$-particle is given by $\mathrm{S}(\mathrm{k}, \mathrm{Z})$ :

$$
\mathrm{S}(\mathrm{k}, \mathrm{Z})=\int_{1+\mathrm{k}}^{\mathrm{W}_{\max }} \mathrm{n}_{\text {cor }}\left(\mathrm{W}_{\mathrm{e}}^{\prime}, \mathrm{k}, \mathrm{Z}\right) \mathrm{P}\left(\mathrm{W}_{\mathrm{e}}^{\prime}\right) \mathrm{dW}_{\mathrm{e}}^{\prime}
$$

Here $\mathrm{P}\left(\mathrm{W}_{\mathrm{e}}{ }^{\prime}\right) \mathrm{dW}_{\mathrm{e}}{ }^{\prime}$ is the $\beta$-spectrum of the ${ }^{45} \mathrm{Ca}$ under study. In the present measurement, the $\beta$-spectrum given by Macklin et al. [16] was used.
The bremsstrahlung photon yield $\mathrm{T}$ for the target, with $\mathrm{k}_{\min }$ and $\mathrm{k}_{\max }$ as the lower and upper limit of photon energy of the bremsstrahlung spectrum, respectively is given by:

$$
\mathrm{T}=\int_{\mathrm{k}_{\min }}^{\mathrm{k}_{\max }} \mathrm{S}(\mathrm{k}, \mathrm{Z}) \mathrm{dk}
$$

Computer programs are written to calculate the bremsstrahlung spectral photon distribution in terms of the number of photons of energy $\mathrm{k}$ per unit $\mathrm{m}_{0} \mathrm{c}^{2}$ per $\beta$-disintegration. The experimental and theoretical results were compared in terms of the number of photons of energy $\mathrm{k}$ per $\mathrm{m}_{0} \mathrm{c}^{2}$ per unit total photon yield. This method is effective to remove the uncertainties associated with the source strength. The results reported here was better accuracy over our previous studies reported in $[6,7]$ due to the addition of thickness of beryllium window and silicon chip in the calculation of bremsstrahlung.

\section{EXPERIMENTAL}

A $\gamma$-ray source of ${ }^{133} \mathrm{Ba}$ and the emitted $\mathrm{K} \mathrm{X}$-rays of Ti, $\mathrm{Cu}, \mathrm{Mo}$ and $\mathrm{Sn}$ materials were used for calibration and determining the detector parameters of the X-PIPS Si(Li) detector. The experimental arrangement is shown in Fig. 1, consisting of X-PIPS (Canberra makes) Si(Li) detector shielded with a lead and further connected to spectroscopy amplifier and multichannel analyzer, was used to calculate the various parameters of the detector. $\mathrm{A}^{45} \mathrm{Ca} \beta$-source having activity $0.5 \mathrm{mCi}$ was used for the present measurements to measure the bremsstrahlung photon distributions in $\mathrm{Al}, \mathrm{Cu}, \mathrm{Sn}$ and $\mathrm{Pb}$ targets produced by beta particles of ${ }^{45} \mathrm{Ca}$ in the photon energy region of 5-30 keV. The detail about the experimental arrangement has already given elsewhere [6].

A $\beta$-stopper technique was used to eliminate the contribution of internal bremsstrahlung (IB), bremsstrahlung generated in the source material and room background to obtain the correct information regarding the bremsstrahlung produced in the target material. The details about this technique have already been mentioned in elsewhere [6]. Targets of $\mathrm{Al}(91$ $\left.\mathrm{mg} \mathrm{cm}{ }^{-2}\right), \mathrm{Cu}\left(91 \mathrm{mg} \mathrm{cm}^{-2}\right), \mathrm{Sn}\left(94 \mathrm{mg} \mathrm{cm}^{-2}\right)$ and $\mathrm{Pb}(93 \mathrm{mg}$ $\mathrm{cm}^{-2}$ ) were used in the present measurements. After calibrating the spectrometer, two sets of measurement were taken for a time interval of 300000 second each to improve the statistics of the data. The statistical accuracy of the data was better than $1 \%$ at the whole of the photon energy region of $5-30 \mathrm{keV}$. These measurements were taken with the targets of $\mathrm{Al}, \mathrm{Cu}, \mathrm{Sn}$ and $\mathrm{Pb}$ for ${ }^{45} \mathrm{Ca} \beta$-particles, to obtain the spectral photon bremsstrahlung distributions.

The measured experimental bremsstrahlung spectra taken in the common channel width of $0.5 \mathrm{keV}$ were converted into a true spectrum by applying the correction due to absorption of bremsstrahlung photons, electron backscattering correction and detector efficiency correction. The correction of the self absorption of bremsstrahlung photons in air, target thickness and the Perspex $\beta$-stopper was applied by using the mass attenuation coefficients recently tabulated by Chantler et al. [15]. The values of electron backscattering factor $\mathrm{R}$ [13] was also incorporated in the measured bremsstrahlung spectra. The corrected experimental bremsstrahlung spectra were converted into the number of photons of energy $\mathrm{k}$ per unit $\mathrm{m}_{0} \mathrm{c}^{2}$ per unit 


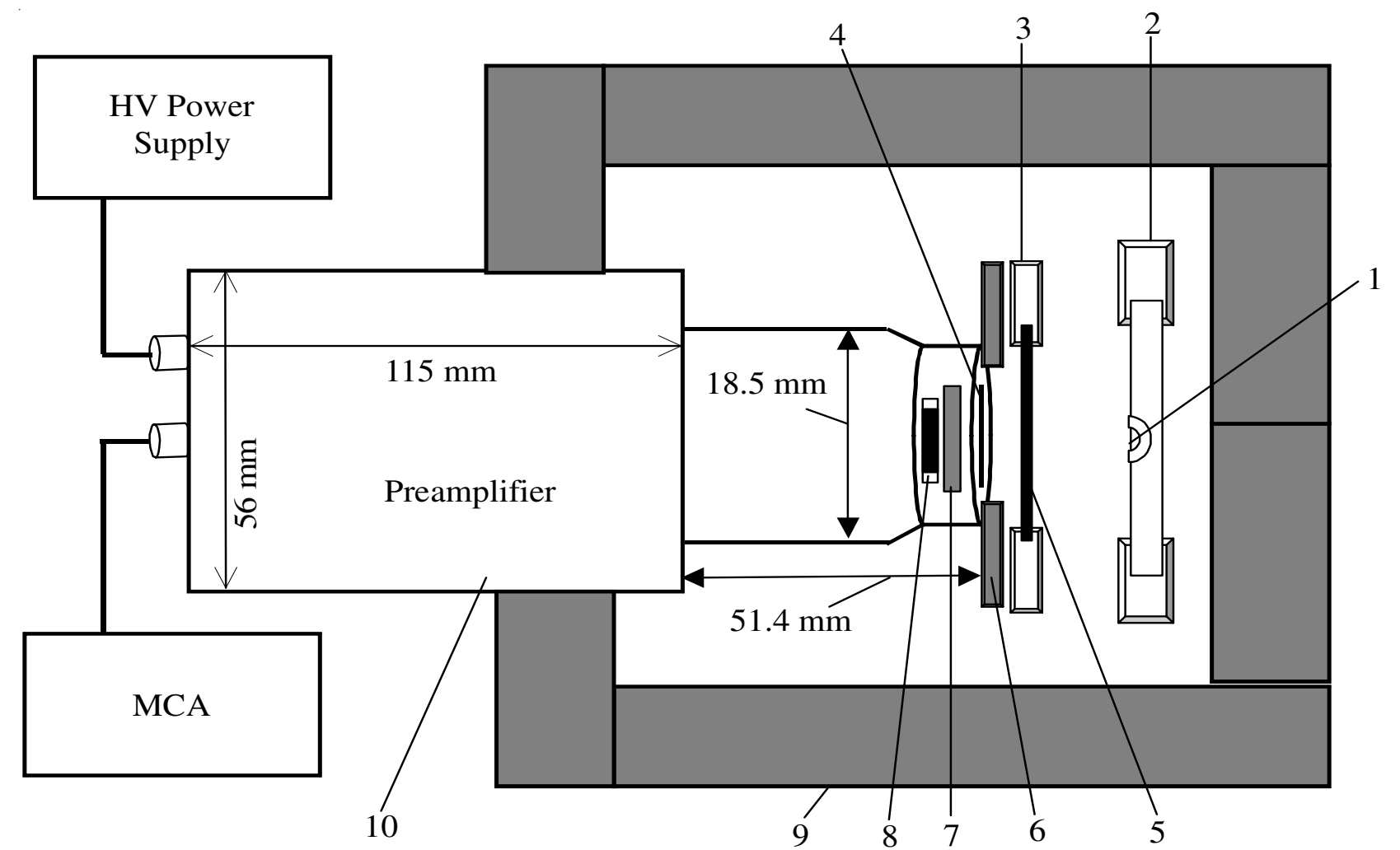

Fig. 1. Experimental arrangement [1. Source position; 2. Source holder; 3. Target holder; 4. Be window; 5. Target; 6. Lead collimator; 7. Detector collimator; 8. Si(Li) chip; 9. Shielding lead bricks; 10. X-PIPS Si(Li) detector

of total photon yield by dividing them by the values of the total photon yields in the target materials. The experimental

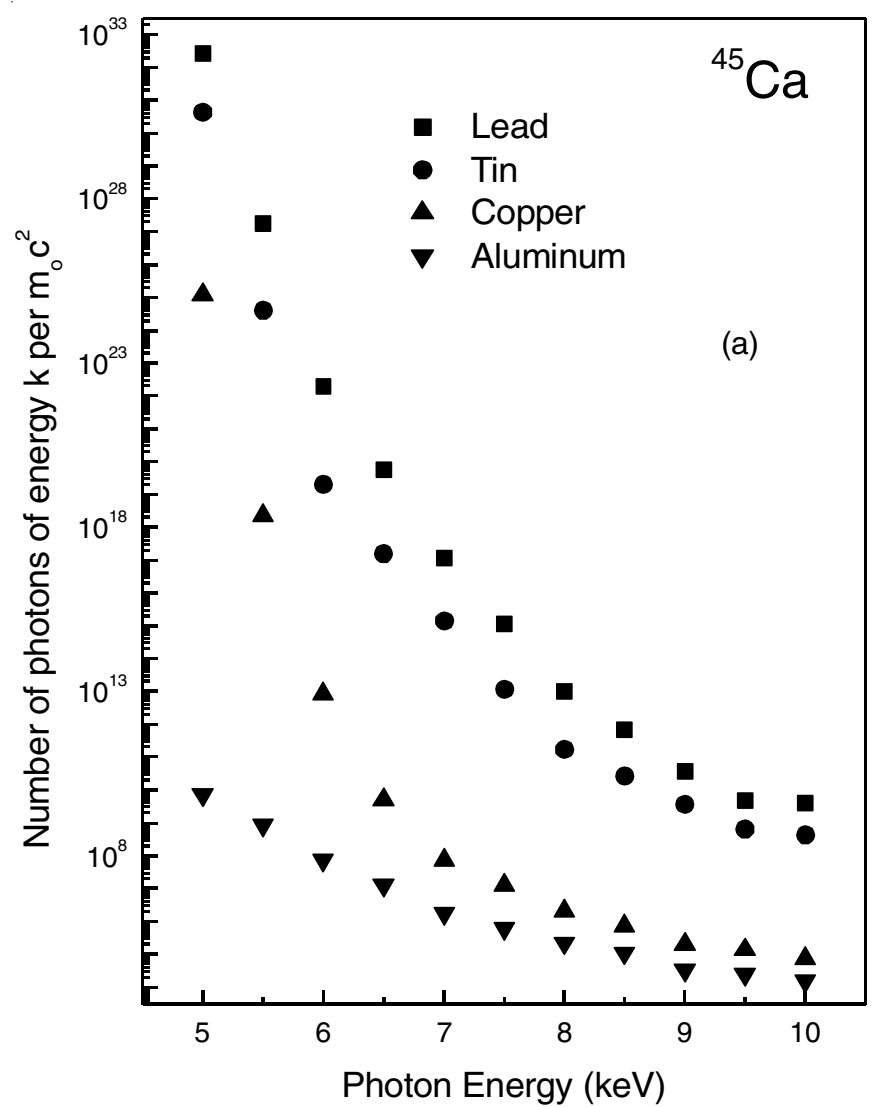

results for $\mathrm{Al}, \mathrm{Cu}, \mathrm{Sn}$ and $\mathrm{Pb}$ targets for ${ }^{45} \mathrm{Ca} \beta$-particles are shown in Fig. 2(a,b).

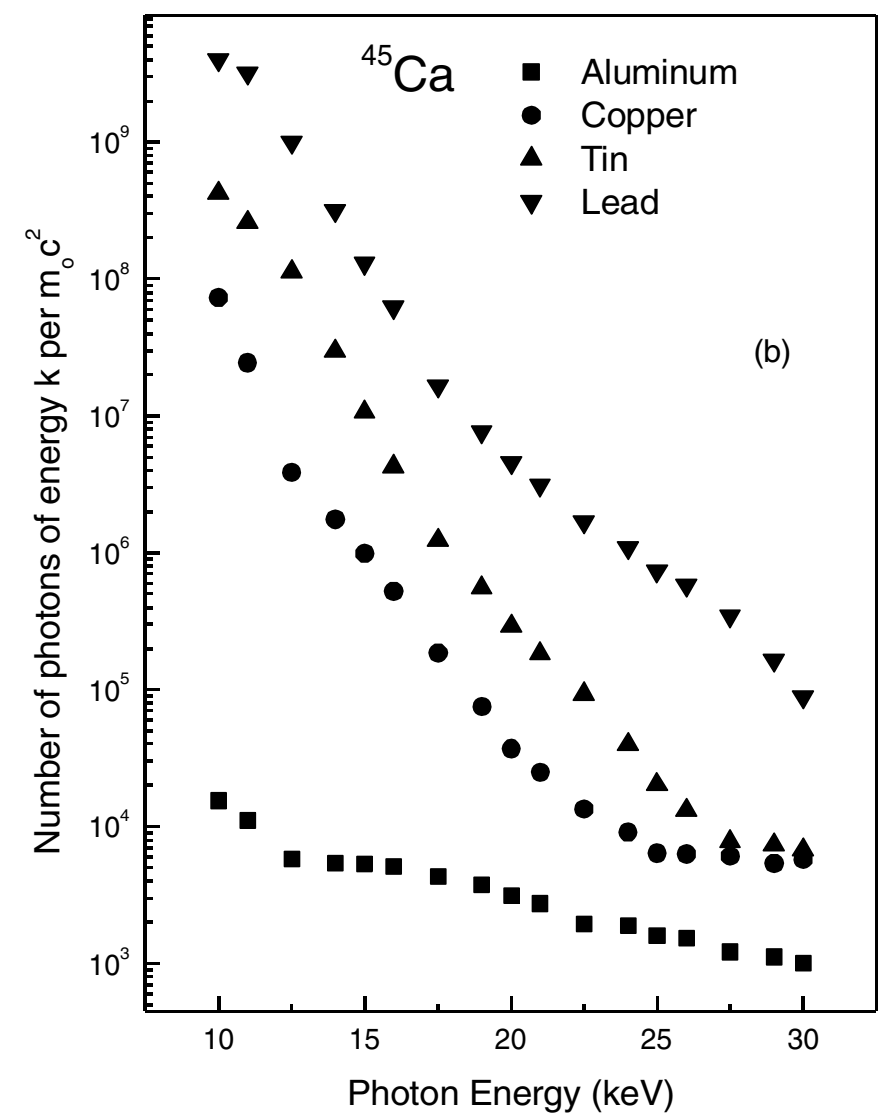

Fig. 2(a-b). Experimental bremsstrahlung spectral distributions in terms of number of photons of energy $\mathrm{k}$ per $\mathrm{m}_{\mathrm{o}} \mathrm{c}^{2}$ versus photon energy $(\mathrm{k})$ for ${ }^{45} \mathrm{Ca}$ beta particles (end point energy $=258 \mathrm{keV}$ ) 
The errors in the present measurement are mainly due to the counting statistics, full energy detection efficiency of the detector, electron backscattering and attenuation of bremsstrahlung photons in the target materials. The uncertainty in the geometrical full-energy peak detector efficiency was not more than $3 \%$. This is due to the uncertainty in the measurement of photo - fractions, which were uncertain of the amount of 1$2 \%$. The uncertainty in the mass attenuation coefficient used in the correction of self absorption of photon in air, target thickness and Perspex beta stopper was less than $1 \%$, except in the near edge regions where uncertainties were higher as reported in the tabulations by Chantler et al. [15]. The uncertainties in the values of the electron backscattering factor $\mathrm{R}$ were less than $1 \%$. The overall uncertainties in the present measurement were estimated to be less than $10 \%$ in the entire photon energy region.

\section{RESULTS AND DISCUSSION}

The percentage energy resolution $\mathrm{R}$ of X-PIPS $\mathrm{Si}(\mathrm{Li})$ detector is defined as:

$$
\mathrm{R}=\frac{\text { Full width at half maximum }}{\text { Pulse height }} \times 100
$$

The energy resolution of X-PIPS $\mathrm{Si}(\mathrm{Li})$ detector is found to be $\leq 190 \mathrm{eV}$ at photon energy $5.9 \mathrm{keV}$.

The photo-fraction $f(k)$ is defined as the ratio of the number of counts under the full energy peak to the total number of counts under the total height spectrum (corrected for X-rays and backscattered peaks). The values of photo-fraction $\mathrm{f}(\mathrm{k})$ of X-PIPS detector were determined by using the ${ }^{133} \mathrm{Ba}$ source and $\mathrm{KX}$-rays peaks of $\mathrm{Ti}, \mathrm{Cu}, \mathrm{Mo}$ and $\mathrm{Sn}$ target materials. The plot of photo-fraction $\mathrm{f}(\mathrm{k})$ values at different photon energies are given in Fig. 3.

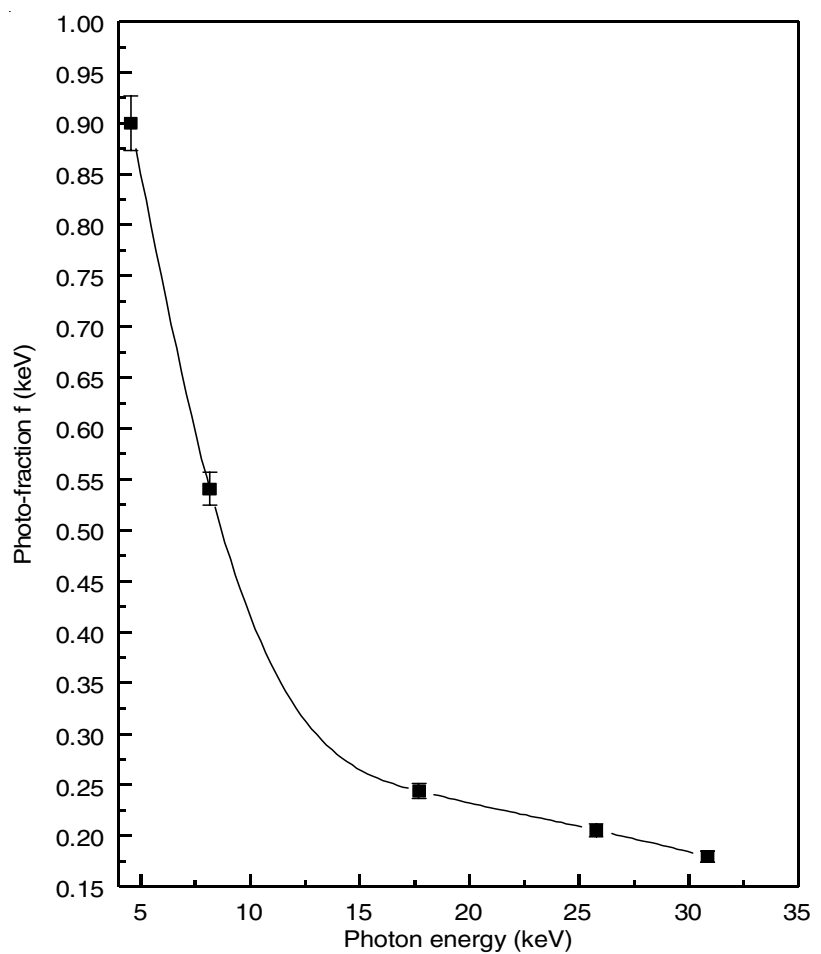

Fig. 3. Variation of Photo-fraction $\mathrm{f}(\mathrm{k})$ versus photon energy $(\mathrm{k})$ of $\mathrm{X}$ PIPS Si(Li) detector
The geometrical full energy peak detector efficiency of $\mathrm{X}$-PIPS $\mathrm{Si}(\mathrm{Li})$ detector is given by the product of the photofraction values $\mathrm{f}(\mathrm{k})$ and the intrinsic efficiency of the detector of particular photon energy. The intrinsic efficiency I $(\mathrm{k})$ of the X-PIPS Si(Li) detectors is varying from $97 \%$ to $15 \%$ at 5 $\mathrm{keV}$ and $30 \mathrm{keV}$ photon energies, respectively. The plot of intrinsic efficiency of X-PIPS $\mathrm{Si}(\mathrm{Li})$ detector is shown in Fig. 4. The geometrical full energy detector efficiency of X-PIPS detector was determined by taking the product of $\mathrm{I}(\mathrm{k}) \times \mathrm{f}(\mathrm{k})$ at particular photon energy. The plot of geometrical full energy peak detector efficiency of X-PIPS $\mathrm{Si}(\mathrm{Li})$ detector versus photon energy is shown in Fig. 5. The geometrical full energy peak detector efficiency is varying from $95 \%$ to $5 \%$ at 5 and $30 \mathrm{keV}$ photon energies, respectively.

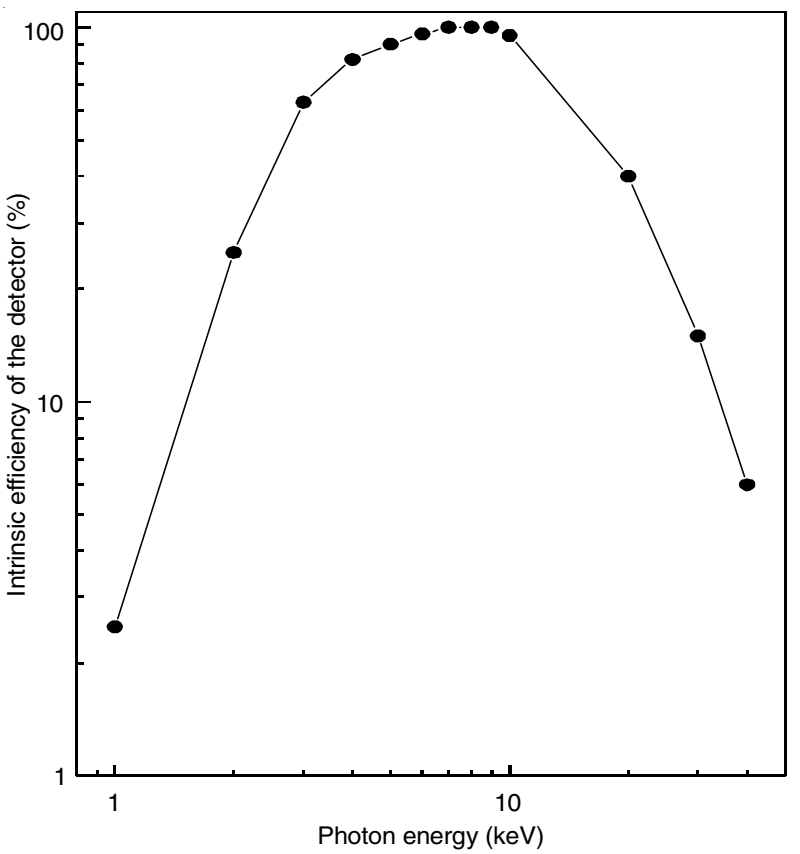

Fig. 4. Percentage Intrinsic efficiency I (k) of X-PIPS Si(Li) detector

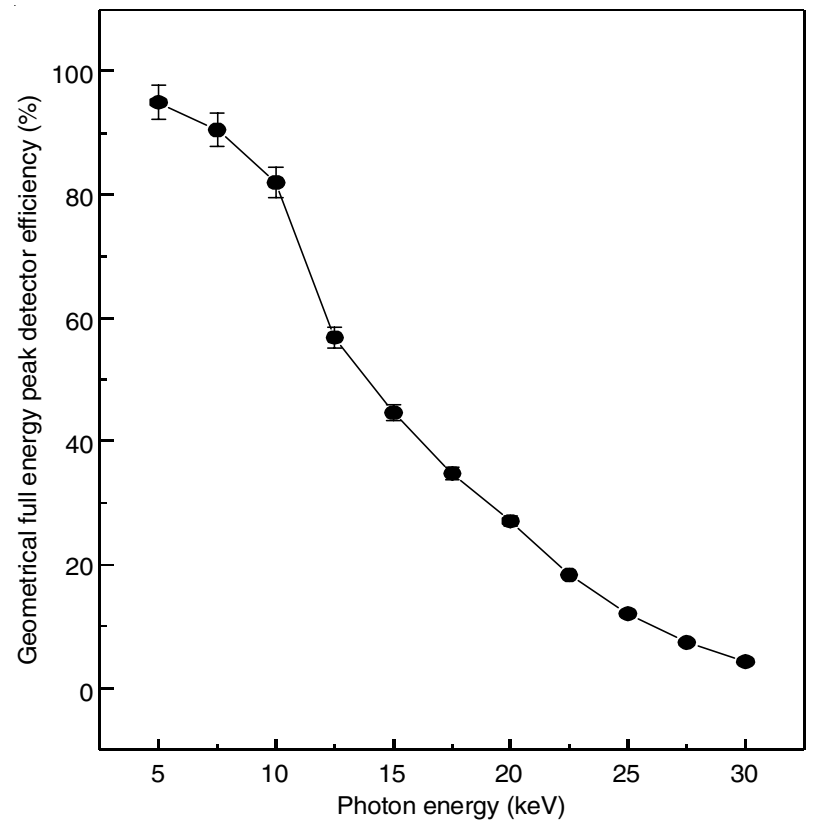

Fig. 5. Percentage Geometrical full energy peak detector efficiency of XPIPS $\mathrm{Si}(\mathrm{Li})$ detector versus photon energy $(\mathrm{k})$ 

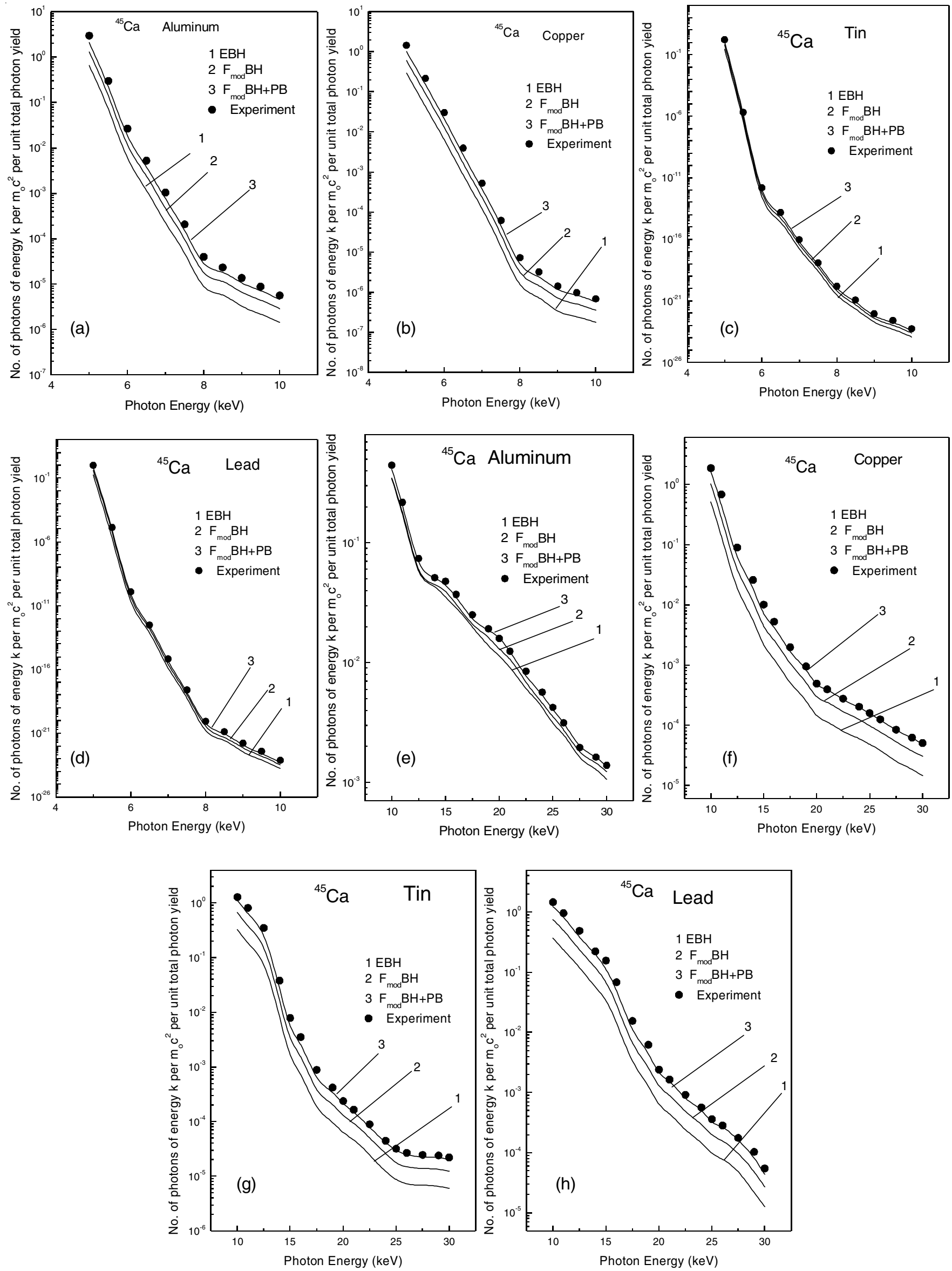

Fig. 6(a-h). Plots of number of bremsstrahlung photons of energy per unit total photon yield versus photon energy (keV) for $\mathrm{Al}, \mathrm{Cu}, \mathrm{Sn}$ and $\mathrm{Pb}$ targets for ${ }^{45} \mathrm{Ca}$ beta particles (errors are lying within the experimental points) 
The results of experimentally measured bremsstrahlung spectral photon distributions for the targets of $\mathrm{Al}, \mathrm{Cu}, \mathrm{Sn}$ and $\mathrm{Pb}$ produced by ${ }^{45} \mathrm{Ca} \beta$-particles, were compared with the theoretical bremsstrahlung spectral photon distributions obtained from Elwert corrected (non relativistic) Bethe-Heitler theory (EBH), modified Elwert factor (relativistic) BetheHeitler theory $\left(\mathrm{F}_{\bmod } \mathrm{BH}\right)$ describes ordinary bremsstrahlung and modified Elwert factor (relativistic) Bethe-Heitler theory $\left(\mathrm{F}_{\bmod } \mathrm{BH}+\mathrm{PB}\right)$ which describes bremsstrahlung spectrum in the photon energy region of $5-30 \mathrm{keV}$. In the photon energy region of $5-10 \mathrm{keV}$, the number of photons emitted is much larger than the number of photons in the energy region of 10$30 \mathrm{keV}$. Therefore, the study of ordinary bremsstrahlung and bremsstrahlung in thick targets produced by beta emitters is reported separately in the photon energy region of $5-10 \mathrm{keV}$ and $10-30 \mathrm{keV}$ to explain the results in a more systematic manner. The plots of the number of photons of energy $\mathrm{k}$ per $\mathrm{m}_{0} \mathrm{c}^{2}$ per unit total photon yield for $\mathrm{Al}, \mathrm{Cu}, \mathrm{Sn}$ and $\mathrm{Pb}$ targets are given in the Fig. 6(a-h).

It has been found that the experimental results are showing better agreement with the theoretical bremsstrahlung spectral photon distributions obtained from $\mathrm{F}_{\bmod } \mathrm{BH}+\mathrm{PB}$ theory. The experimental results show deviation from $\mathrm{EBH}$ and $\mathrm{F}_{\bmod } \mathrm{BH}$ theories. These deviations are found to be varying from 12 to $29 \%$ of $\mathrm{Sn}$ and $\mathrm{Pb}$ targets, respectively from $\mathrm{EBH}$ and $\mathrm{F}_{\bmod } \mathrm{BH}$ theory. However, these deviations are found to be varying between 5 to $23 \%$ in the case of $\mathrm{Al}$ and the deviations are found to be varying between 10 to $27 \%$ of $\mathrm{Cu}$ target throughout the studied photon energy region. These results clearly indicate that the polarization bremsstrahlung contributions must be taken into account while studying the bremsstrahlung spectral photon distribution in thick metallic targets, produced by beta particles in ${ }^{45} \mathrm{Ca}$ having energy range $0-258 \mathrm{keV}$ in the studied photon energy region of 5-30 keV. It has been observed that for high $\mathrm{Z}$ elements the contribution of polarization bremsstrahlung into ordinary bremsstrahlung is higher as compared to the low $\mathrm{Z}$ elements.

\section{CONFLICT OF INTEREST}

The author declares that there is no conflict of interests regarding the publication of this article.

\section{REFERENCES}

1. J.A. Gray, Proc. R. Soc. Math. Phys. Eng. Sci., 85, 131 (1911); https://doi.org/10.1098/rspa.1911.0027.

2. J.A. Gray, Proc. R. Soc., Math. Phys. Eng. Sci., 86, 513 (1912); https://doi.org/10.1098/rspa.1912.0043.

3. J. Hamilton, L. Langer and W. Smith, Phys. Rev., 112, 2010 (1958); https://doi.org/10.1103/PhysRev.112.2010.

4. A.S. Dhaliwal, M.S. Powar and M. Singh, Phys. Rev. A, 48, 1308 (1993); https://doi.org/10.1103/PhysRevA.48.1308.

5. S. Portillo and C.A. Quarles, Phys. Rev. Lett., 91, 173201 (2003); https://doi.org/10.1103/PhysRevLett.91.173201.

6. T. Singh, K.S. Kahlon and A.S. Dhaliwal, J. Phys. At. Mol. Opt. Phys., 41, 235001 (2008); https://doi.org/10.1088/0953-4075/41/23/235001.

7. T. Singh, K.S. Kahlon and A.S. Dhaliwal, Nucl. Instrum. Methods Phys. Res. B, 267, 737 (2009);

https://doi.org/10.1016/j.nimb.2009.01.009.

8. V. Buimistrov and L. Trakhtenberg, SZh. Eksp. Teor. Fiz., 42, 54 (1975).

9. M.Ya. Amusya, M.Yu. Kuchiev, A.V. Korol and A.V. Solov'yov, Zh. Eksp. Teor. Fiz., 61, 224 (1985).

10. A.V. Korol, O.I. Obolensky and A.V. Solov'yov, J. Phys. At. Mol. Opt. Phys., 31, 5347 (1998); https://doi.org/10.1088/0953-4075/31/24/015.

11. N.B. Avdonina and R.H. Pratt, J. Phys. At. Mol. Opt. Phys., 32, 4261 (1999); https://doi.org/10.1088/0953-4075/32/17/310.

12. H. Bethe and W. Heitler, Proc. R. Soc. Lond. A Math. Phys. Sci., 146, 83 (1934); https://doi.org/10.1098/rspa.1934.0140.

13. M. Semaan and C.A. Quarles, X Ray Spectrom., 30, 37 (2001); https://doi.org/10.1002/xrs.465.

14. M.J. Berger and S.M. Seltzer, NASA SP 3012 (1964); Current Tabulations on the Web: Program ESTAR; http://Physics.nist.gov./PhysRefData/Star/Text/ESTAR.Html.

15. C.T. Chantler, K. Olsen, R.A. Dragoset, J. Chang, A.R. Kishore, S.A. Kotochigova and D.S. Zucker, X-Ray Form Factor, Attenuation and Scattering Tables (Version 2.1), National Institute of Standards and Technology, Gaithersburg, MD, Germany (2005).

16. P. Macklin, L. Feldman, L. Lidofsky and C. S. Wu, Phys. Rev., 77, 137 (1950); https://doi.org/10.1103/PhysRev.77.137. 